\title{
Effect of Delivered Dosage of Cigarette Smoke Toxins on the Levels of Urinary Biomarkers of Exposure
}

\author{
Assieh A. Melikian, ${ }^{1}$ Mirjana V. Djordjevic, ${ }^{2}$ Shuquan Chen, ${ }^{3}$ John Richie, Jr., ${ }^{4}$ \\ and Steven D. Stellman ${ }^{5}$ \\ ${ }^{1}$ Department of Environmental Medicine, New York University School of Medicine, Tuxedo, New York; ${ }^{2}$ National Cancer Institute, \\ Tobacco Control Research Branch, Bethesda, Maryland; ${ }^{3}$ Former American Health Foundation, Valhalla, New York; \\ ${ }^{4}$ Penn State University, Hershey Medical Center, Hershey, Pennsylvania; and ${ }^{5}$ Department of Epidemiology, \\ Mailman School of Public Health, Columbia University, New York, New York
}

\begin{abstract}
Urinary metabolites of tobacco smoke toxins are often used as biomarkers for the evaluation of active and passive exposure to cigarette smoke toxins. In a study of healthy smokers, we investigated concentrations of urinary biomarkers in relation to concentrations of selected toxins in mainstream cigarette smoke as determined by machine smoking of cigarettes in a manner that mimics an individual's smoking behavior (topography). Concentrations of nicotine, 4-(methylnitrosamino)-1-(3-pyridyl)-1-butanone, and benzo(a)pyrene, in mainstream smoke determined under human smoking conditions, and their urinary metabolites cotinine, 4-(methylnitrosamino)-1-(3-pyridyl)-1-butanol, and 1-hydroxypyrene were established for 257 individuals who smoked low-yield (0.1-0.8 mg Federal Trade Commission nicotine/cigarette; mean, 0.66; $n=87)$, medium-yield (0.9-1.2 $\mathrm{mg}$ nicotine/ cigarette; mean, 1.1; $n=109$ ), and high-yield cigarettes (nicotine, $>1.3 \mathrm{mg}$ nicotine/cigarette; mean, $1.41 ; n=61$ ). Levels of urinary metabolites expressed per unit of delivered parent compounds decreased with increased smoke emissions. In smokers of low-, medium-, and high-yield cigarettes, the respective cotinine ( $\mathrm{ng} / \mathrm{mg}$ creatinine)-to-nicotine $(\mathrm{mg} / \mathrm{d})$

ratios were $89.4,77.8$, and 57.1 (low versus high; $P=0.06$ ); the 4-(methylnitrosamino)-1-(3-pyridyl)-1-butanol (pmol/mg creatinine)-to-4-(methylnitrosamino)-1-(3-pyridyl)-1-butanone (ng/d) ratios were $0.81,0.55$, and 0.57 (low versus high; $P=0.05)$; and the 1-hydroxypyrene (pg/mg creatinine)to-benzo $(a)$ pyrene $(\mathrm{ng} / \mathrm{d})$ ratios were $1.55,1.13$, and 0.97 (low versus high; $P=0.008$ ). Similarly, means of cotinine per unit of delivered nicotine in smokers who consumed $<20$ cigarettes per day was 3.5 -fold higher than in those who smoked $>20$ cigarettes per day. Likewise, a negative correlation was observed between cotinine-to-nicotine ratios and delivered doses of nicotine in subgroups of smokers who used the identical brand of cigarette, namely a filter tip-vented Marlboro $(r=-0.59)$, which is a popular brand among EuroAmericans, and Newport $(r=-0.37)$, a menthol-flavored cigarette without filter tip vents that is preferred by AfricanAmericans. Thus, the intensity of the exposures significantly affects the levels of urinary biomarkers of exposure and should be taken into account in the evaluation of human exposure to cigarette smoke toxins. (Cancer Epidemiol Biomarkers Prev 2007;16(7):1408-15)
\end{abstract}

\section{Introduction}

The association of cigarette smoking with the risk for various human cancers, chronic obstructive pulmonary disease, and cardiovascular disease is well established $(1,2)$. Epidemiologic studies have also shown a dose-response relationship between cigarette smoking and adverse health outcomes based on smoking intensity and duration of smoking $(1,2)$. Thus, one would anticipate a relationship between exposure to tobacco toxins and disease risk.

Cigarette smoke is a highly complex matrix with almost 4,800 constituents identified (1). More than sixty of the identified compounds, including polynuclear aromatic hydrocarbons (PAH) and tobacco-specific $N$-nitrosamines, are classified as carcinogens by the IARC based on evidence in humans and/or animals (1). The delivered dosage of tobacco smoke compounds is influenced not only by cigarette composition and design but also by many smoker-dependent variables, such as number of cigarettes smoked per day, puffing patterns, blocking of filter vents, and length of cigarette smoked (3-5). The delivery of specific toxic constit-

E-mail: Melikian@med.nyu.edu

Copyright (C) 2007 American Association for Cancer Research.

doi:10.1158/1055-9965.EPI-06-1097

uents from each cigarette smoked by an individual can be determined by machine smoking that individual's particular brand of cigarette under conditions that mimic their specific puffing pattern, butt lengths, and blocking of the filter vents $(6,7)$. Puffing parameters (topography) are usually assessed using a computer-assisted flow transducer that determines the flow of smoke from a lit cigarette as it is smoked $(6,7)$. Internal exposure can also be assessed in individual smokers by measuring the sum of toxins and their metabolites in appropriate biological fluids. However, although urinary metabolites are commonly used to evaluate smokers' exposure to nicotine and carcinogens, interpretation of these biomarker levels are complicated by interindividual differences in metabolism (8-12). The availability of more than one thousand brands on the market [e.g., 1,294 brands in the United States in 1998; Federal Trade Commission (FTC) report issued in 2000], the variety of ways in which smokers use the same or different products, and the influence of gender, race, and age on metabolism of toxins challenge the accurate evaluation of smokers' exposure to cigarette smoke toxins.

The goal of this study is to examine the relationships between delivered dosages of smoke constituents, such as nicotine and select carcinogens, determined using actual human smoking conditions and levels of corresponding urinary metabolites in smokers. The effect of cigarette brand and associated yield (FTC) of nicotine and tar on these relationships will also be examined. To this end, we measured the delivery of nicotine and select cigarette smoke constituents among adult smokers as described earlier $(6,7)$ and determined the levels of corresponding metabolites in urine. 
Specifically, we quantified nicotine, 4-(methylnitrosamino)-1(3-pyridyl)-1-butanone (NNK), and benzo(a)pyrene ( $\mathrm{BaP}$; as a marker of exposure to $\mathrm{PAH}$ ) in the mainstream smoke condensate generated by machine smoking of each individual's cigarettes under conditions that reflect that individual's smoking pattern. Levels of urinary cotinine were determined for nicotine exposure, and 4-(methylnitrosamino)-1-(3-pyridyl)-1-butanol (NNAL) was used as a marker for NNK, whereas 1-hydroxypyrene (1-OH-P) was quantified as a biomarker for PAH exposures.

\section{Materials and Methods}

Study Subjects and Sample Collection. In this communitybased study, adult volunteers between 18 and 59 years of age and in good general health were recruited from the local community as described previously $(7,13)$. These volunteers were screened by telephone to determine whether they met the following specific criteria: they had to have smoked at least 10 cigarettes of their current brand daily for at least 1 year and had to be in good health, without a history of any tobaccorelated disease, and without any unstable medical condition. Also they had to be free from psychotropic medications and without any psychiatric diagnosis at the time of study. They were not eligible if they were using any tobacco- or nicotinecontaining products other than cigarettes for at least 3 months before the study. Pregnant and nursing women were excluded from the study. To our knowledge, those who enrolled in this study were not participating to any tobacco cessation program or seeking any type of treatment.

Subjects enrolled in the study voluntarily in response to newspaper advertisements in Westchester County, New York, and remunerated for their participation. All subjects gave written consent, and the study was approved by the American Health Foundation's Institutional Human Subjects Review Committee in accordance with assurances filed with and approved by the U.S. Department of Health and Human Services.

A telephone interview determined initial eligibility. Eligible volunteers were enrolled and received detailed information about study goals and procedures. On signing consent, they were asked to collect cigarette butts for a total of 4 days before their visit. The butts were used to validate the subject's selfreported number of cigarettes smoked per day, to assess the average length of each cigarette smoked, and to evaluate whether blocking of the air vents of filter tips had occurred during smoking. A trained interviewer administered a comprehensive questionnaire to obtain information on smoking history, namely the age at onset of smoking, the quantity and type of cigarettes and number of years smoked, occupational exposure, family medical history, diet and other lifestyle factors, as well as nicotine dependence using the Fagerstrom questionnaire. During the interview, volunteers disclosed the brand of cigarettes they smoked, type of pack (hard or soft), and whether they smoked mentholated or nonmentholated cigarettes. Detailed cigarette brand information was obtained to assign the correct FTC smoke yield because several varieties with different FTC smoke yields are marketed under the same brand name.

Most smoking measurements were carried out between 10:00 a.m. and 1:00 p.m. A single urine sample was collected from each smoker about the same time of the day after they smoked three or four cigarettes for smoking topography measurements (7).

About half of the subjects were recruited within years 2001 and 2002 and the other major recruitment was in the years 1997 and 1998. Each smoker's cigarettes were purchased at the same time the urine sample was collected.

Measurement of Toxic and Carcinogenic Compounds in Emissions of Mainstream Smoke. The smoking parameters were determined by means of a computer-assisted pressure transducer system as described previously $(6,7)$. Each individual's brand of cigarette was then machine smoked with his or her average smoking parameters, including blocking of the filter tip vents and specific butt length. For determination of each analyte, the smoke particulates from four cigarettes were collected on a Cambridge filter pad and analyzed as described below.

Nicotine content in smoke particulate was analyzed by gas chromatography using a nitrogen phosphorous detector $(6,7$, $14,15)$, NNK concentrations were determined by gas chromatography using a thermal energy analyzer as a detector (gas chromatography with nitrosamine-selective detection; ref. 16), and $\mathrm{BaP}$ was determined by gas chromatography-mass spectrometry (17).

\section{Analysis of Urinary Biomarkers}

Simultaneous Determination of Nonconjugate Urinary 1-OH-P, 1-OH-P Glucuronide Conjugate, and 1-OH-P-Sulfate Conjugate. Free and conjugated 1-OH-P were analyzed by modification of

Table 1. Demographic information for study subjects

\begin{tabular}{|c|c|c|c|c|c|c|c|c|c|}
\hline \multirow[t]{3}{*}{ Variables } & \multicolumn{2}{|c|}{ ALL } & \multicolumn{2}{|c|}{ AA } & \multicolumn{2}{|c|}{ EA } & \multicolumn{3}{|c|}{$P$} \\
\hline & \multirow{2}{*}{$\frac{\text { Female }}{\text { GM }(95 \% \mathrm{CI})}$} & \multirow{2}{*}{$\frac{\text { Male }}{\text { GM }(95 \% \text { CI })}$} & \multirow{2}{*}{$\frac{\text { Female }}{\text { GM }(95 \% \mathrm{CI})}$} & \multirow{2}{*}{$\frac{\text { Male }}{\text { GM }(95 \% \mathrm{CI})}$} & \multirow{2}{*}{$\frac{\text { Female }}{\text { GM }(95 \% \mathrm{CI})}$} & \multirow{2}{*}{$\frac{\text { Male }}{\text { GM }(95 \% \mathrm{CI})}$} & \multicolumn{3}{|c|}{ (Female vs male) } \\
\hline & & & & & & & $\mathrm{ALL}^{*}$ & $\mathrm{AA}^{\dagger}$ & $\mathrm{EA}^{\dagger}$ \\
\hline No. subjects/group ${ }^{\ddagger}$ & 129 & 128 & 57 & 55 & 72 & 73 & & & \\
\hline Age $(y)$ & $33.1(31.2-35.0)$ & $35.0(33.3-36.9)$ & $34.4(31.8-37.3)$ & $36.4(34.0-39.1)$ & $32.1(29.6-34.8)$ & $34.1(31.7-36.6)$ & 0.14 & 0.29 & 0.28 \\
\hline Body mass index & $25.5(24.5-26.5)$ & $26.8(26.1-27.4)$ & $27.6(26.0-29.4)$ & $27.3(26.2-28.4)$ & $24.0(22.9-25.1)$ & $26.4(25.6-27.2)$ & 0.06 & 0.74 & 0.0007 \\
\hline Cigarettes per day & $15.9(14.7-17.1)$ & $16.8(15.5-18.2)$ & $14.3(12.8-16.0)$ & $14.1(12.8-15.6)$ & $17.2(15.6-19.0)$ & $19.1(17.1-21.4)$ & 0.42 & 0.83 & 0.17 \\
\hline $\begin{array}{l}\text { Age at onset of } \\
\text { smoking }(y)\end{array}$ & $15.3(14.7-15.8)$ & $16.4(15.8-17.0)$ & $15.8(14.8-16.8)$ & $16.6(15.8-17.5)$ & $14.9(14.3-15.5)$ & $16.3(15.5-17.1)$ & 0.008 & 0.2 & 0.009 \\
\hline Years smoked & $14.9(13.2-16.9)$ & $15.8(14.0-17.9)$ & $15.7(13.2-18.5)$ & $17.4(14.9-20.4)$ & $14.3(12.0-17.1)$ & $14.8(12.4-17.6)$ & 0.44 & 0.37 & 0.81 \\
\hline Fagerström index & $4.11(3.65-4.62)$ & $4.57(4.12-5.06)$ & $4.12(3.49-4.89)$ & $4.28(3.69-4.93)$ & $4.09(3.46-4.80)$ & $4.80(4.16-5.52)$ & 0.23 & 0.78 & 0.15 \\
\hline $\begin{array}{l}\text { Length of cigarette } \\
\text { smoked (mm) }\end{array}$ & $54.1(52.7-55.5)$ & $53.2(51.7-54.9)$ & $57.3(55.1-59.6)$ & $55.3(52.8-57.9)$ & $51.7(50.1-53.4)$ & $51.8(49.9-53.9)$ & 0.39 & 0.24 & 0.93 \\
\hline Butt length (mm) & 36.3 (35.4-37.2) & $34.3(33.4-35.2)$ & $34.5(33.4-35.5)$ & $33.9(32.4-35.4)$ & $37.8(36.5-39.1)$ & $34.6(33.4-35.8)$ & 0.005 & 0.53 & 0.0005 \\
\hline $\begin{array}{l}\text { Cigarette type: } \\
\text { mentholated }\end{array}$ & $50 \%$ & $50 \%$ & $88 \%$ & $80 \%$ & $21 \%$ & $27 \%$ & & & \\
\hline
\end{tabular}

NOTE: Data are presented in geometric mean and 95\% CI.

Abbreviations: GM, geometric mean; AA, African-Americans; EA, Euro-Americans.

* $P$ values are adjusted for race using ANOVA models.

$\dagger P$ values are based on Student's $t$ test.

$\ddagger$ A total of 257 subjects were included in the analysis, after excluding 5 subjects whose cotinine levels were $<130 \mathrm{ng} / \mathrm{mL}$. 
Table 2. Type and brand of cigarettes smoked by study subjects

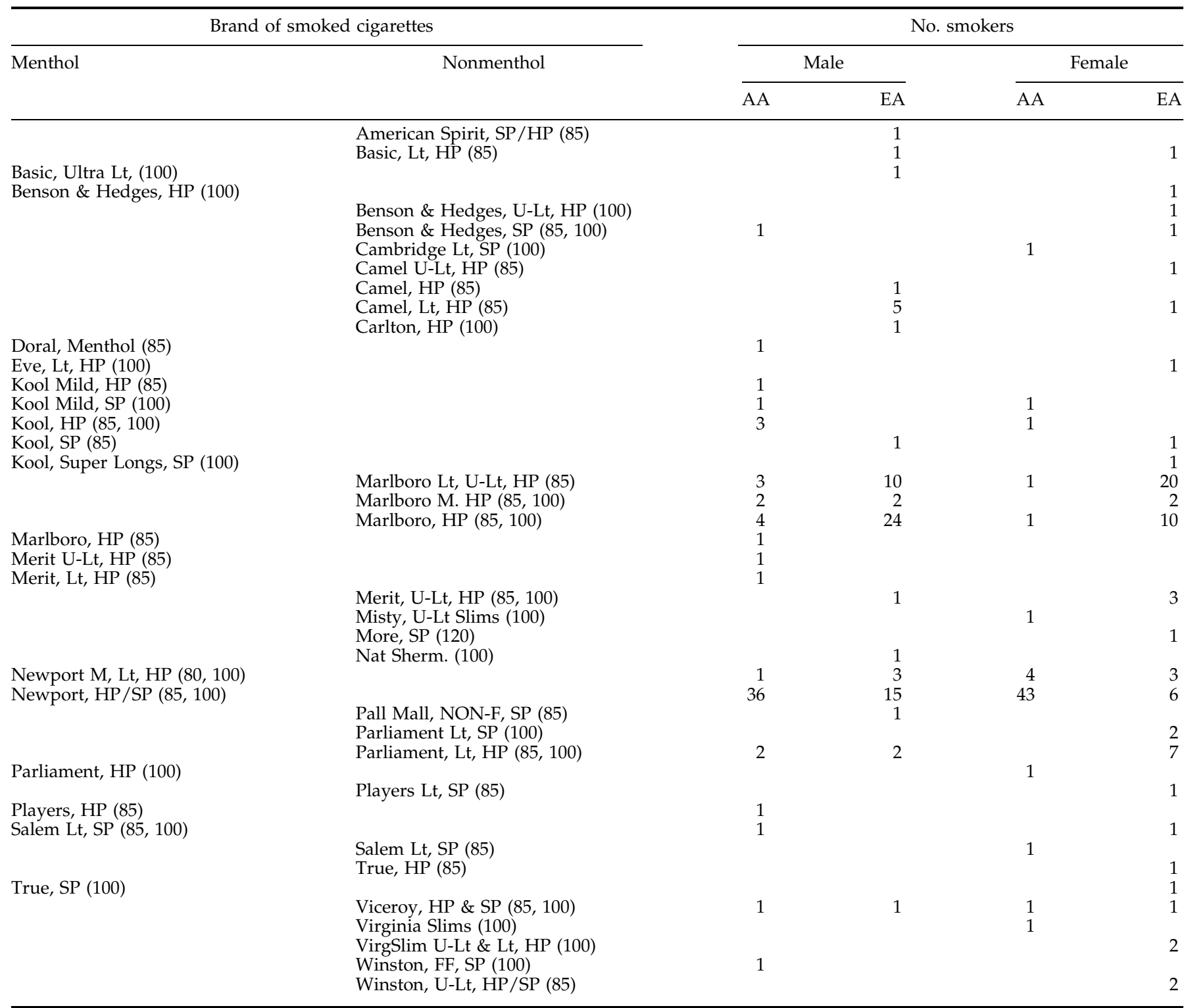

Abbreviations: HP, hard pack; SP, soft pack; U-Lt, ultra light; Lt, light.

a method reported previously $(18,19)$. The procedure is designed to quantify simultaneously 1-OH-P glucuronide (1-OH-P-gluc) conjugate, 1-OH-P-sulfate (1-OH-P-sulf), and free $1-\mathrm{OH}-\mathrm{P}$. In brief, each urine sample $(1.5 \mathrm{~mL})$ was transferred into an autosampler vial, and $150 \mu \mathrm{L}$ DMSO, free of 1-OH-P, obtained from Pierce was added. The mixture was vortexed, and a 100 or $250 \mu \mathrm{L}$ aliquot of each prepared sample was injected and analyzed by high-pressure liquid chromatography with fluorescence detector. The modified high-pressure liquid chromatography system separates watersoluble impurities before elution of the desired analytes of 1-OH-P-gluc conjugate; thus, the urine clean-up step before the high-pressure liquid chromatography analysis is not required. A Zorbox SB-Phenyl column $(250 \times 4.6 \mathrm{~mm}$ ID, $5 \mu \mathrm{m}$; Agilent Technologies) was used at ambient temperature. A gradient elution using $0.01 \mathrm{~mol} / \mathrm{L}$ sodium dihydrogen phosphate buffer ( $\mathrm{pH} 3.5$ ) and acetonitrile was carried out with buffer/acetonitrile (95:5) for $5 \mathrm{~min}$, followed by a gradient from the 95:5 to 50:50 (buffer/acetonitrile) for $35 \mathrm{~min}$, and finally $100 \%$ acetonitrile for $10 \mathrm{~min}$, at a flow rate of $1 \mathrm{~mL} / \mathrm{min}$. Fluorescence was monitored with a Shimatzu RF-10AXL detector with the $\lambda_{\mathrm{EX}}$ fixed at $345 \mathrm{~nm}$ and the $\lambda_{\mathrm{EM}}$ at $385 \mathrm{~nm}$. The 1-OH-P-gluc, 1-OH-P-sulf, and free 1-OH-P were confirmed by cochromatography with standard samples that were obtained from the National Cancer Institute Chemical Carcinogen Reference Standard Repositories. Quantified concentrations of urinary 1-OH-P-gluc and 1-OH-Psulf were converted to $1-\mathrm{OH}-\mathrm{P}$ by calculation and the sum of the three forms as determined based on 1-OH-P was reported (Table 3), and 1-OH-P-gluc was the major $(>90 \%)$ urinary metabolite of pyrene. For checking the high-pressure liquid chromatography column performance and the instrument function, standard samples containing 1-OH-P-gluc, 1-OH-P-sulf, and 1-OH-P were included in the beginning and in the end of each daily batch of high-pressure liquid chromatography analysis with automatic injector. Similarly, blank control samples were analyzed in the middle of each batch to assure that there is no contamination (we found that some of the commercial DMSO contain trace amount of 1-OH-P) and no carryover from one sample to the other. The coefficients of variation for the $1-\mathrm{OH}-\mathrm{P}$ assay were $4.3 \%$, $6.6 \%$, and $4.7 \%$ for $1-\mathrm{OH}-\mathrm{P}$-gluc, $1-\mathrm{OH}-\mathrm{P}$-sulf, and $1-\mathrm{OH}-\mathrm{P}$, respectively, at a concentration of $320 \mathrm{pg} / \mathrm{mL}$ of the analytes $(n=9)$. 
Analyses of Urinary Nonconjugated and Glucuronide Conjugate of NNAL and Cotinine. Urinary NNAL was determined by gas chromatography with nitrosamine-selective detection, using iso-NNAL as an internal standard. NNAL-glucuronide was quantified after hydrolysis to NNAL (20). NNAL and isoNNAL standard samples were purchased from Toronto Research Chemicals, Inc. Cotinine and creatinine were determined as described previously (21).

Statistical Analysis. Outcome parameters (smoking characteristics, cigarette smoke emissions, and urinary biomarkers) were compared between groups using $t$ tests and ANCOVA models to adjust for race and other covariates, such as body mass index. Due to the non-normal distribution of outcomes, all data were log transformed and are presented as geometric means with $95 \%$ confidence intervals $(95 \% \mathrm{CI})$. All tests were considered statistically significant at $P<0.05$.

\section{Results}

Two-hundred and sixty two healthy eligible smokers were recruited. Five subjects were excluded from the study because their urinary cotinine was $<130 \mathrm{ng} / \mathrm{mg}$ creatinine. The subjects were equally distributed with regard to gender and nearly equally with regard to race (56\% Euro-American and $44 \%$ African-American). The demographic characteristics of the smokers are summarized in Table 1. The four gender-ethnic groups were similar in age and years of smoking. Women tended to have begun smoking earlier than men, and EuroAmerican women had significantly lower body mass than Euro-American men. Men had smoked significantly larger portions of their cigarettes (as indicated by shorter average butt lengths) than women (7). Preference for mentholated brands was greater in African-Americans (88\% of females and $80 \%$ of males) than in Euro-Americans (21\% of females and $27 \%$ of males).

The participants in this study smoked 45 different brands of cigarettes, of which three were predominant (Table 2). The menthol-containing, nonventilated Newport brand $(1.4 \mathrm{mg}$ FTC nicotine/cigarette) was the type of cigarette smoked most often by African-Americans (70.5\%), whereas 23.4\% of EuroAmericans smoked nonmentholated, filter-ventilated Marlboro (1.1 mg FTC nicotine/cigarette) and $21.7 \%$ of Euro-American smoked Marlboro light (0.8 mg FTC nicotine/cigarette). These three brands combined were preferred by two thirds of all smokers.

Comparison of the Levels of Urinary Metabolites Excreted in Smokers who Smoked Low-, Medium-, and High-Yield Cigarettes. Classification of cigarette type as "low yield" (0.1$0.8 \mathrm{mg}$ nicotine/cigarette), "medium yield" (0.9-1.2 mg nicotine/cigarette), and "high yield" (1.3-1.9 mg nicotine/ cigarette) was based on FTC nicotine levels determined from the most recent available data (FTC, issued in 2000). The geometric means of nicotine, $\mathrm{NNK}$, and $\mathrm{BaP}$ emissions per cigarette and per day obtained under human smoking conditions and levels of urinary cotinine, total NNAL, and 1$\mathrm{OH}-\mathrm{P}$ (before and after adjusting to delivered doses of parent compounds) in low-, medium-, and high-yield cigarette smokers are summarized in Table 3 . The delivery of nicotine in mainstream smoke under human smoking conditions, measured either as $\mathrm{mg} /$ cigarette or $\mathrm{mg} / \mathrm{d}$, followed the same trend as FTC-reported nicotine yields $(P<0.002)$. A similar trend was observed for $\mathrm{BaP}$ emission, although the human smoking condition levels of $\mathrm{BaP}$ in smokers of medium- and high-yield cigarettes were not significantly different. The average human smoking condition yield of NNK was higher in smokers of medium-yield group (most of whom smoked Marlboros) compared with smokers of high-yield cigarettes (predominantly smokers of Newports; $P>0.02$ ). The yield of NNK in Newport cigarettes is less than in Marlboros (data not shown).

There were no significant differences in levels of cotinine, NNAL, and 1-OH-P among the groups smoking low-, mediumor high-yield cigarettes, except that levels of cotinine (unadjusted for dosage) were significantly lower $(P=0.003)$ in those smoking low-yield versus medium-yield cigarette. However,

Table 3. Emissions of toxins from cigarettes in low-, medium-, and high-yield cigarette smokers and excretion of toxic metabolites in smokers' urine before and after adjusting for delivered doses of parent compounds in mainstream cigarette smoke

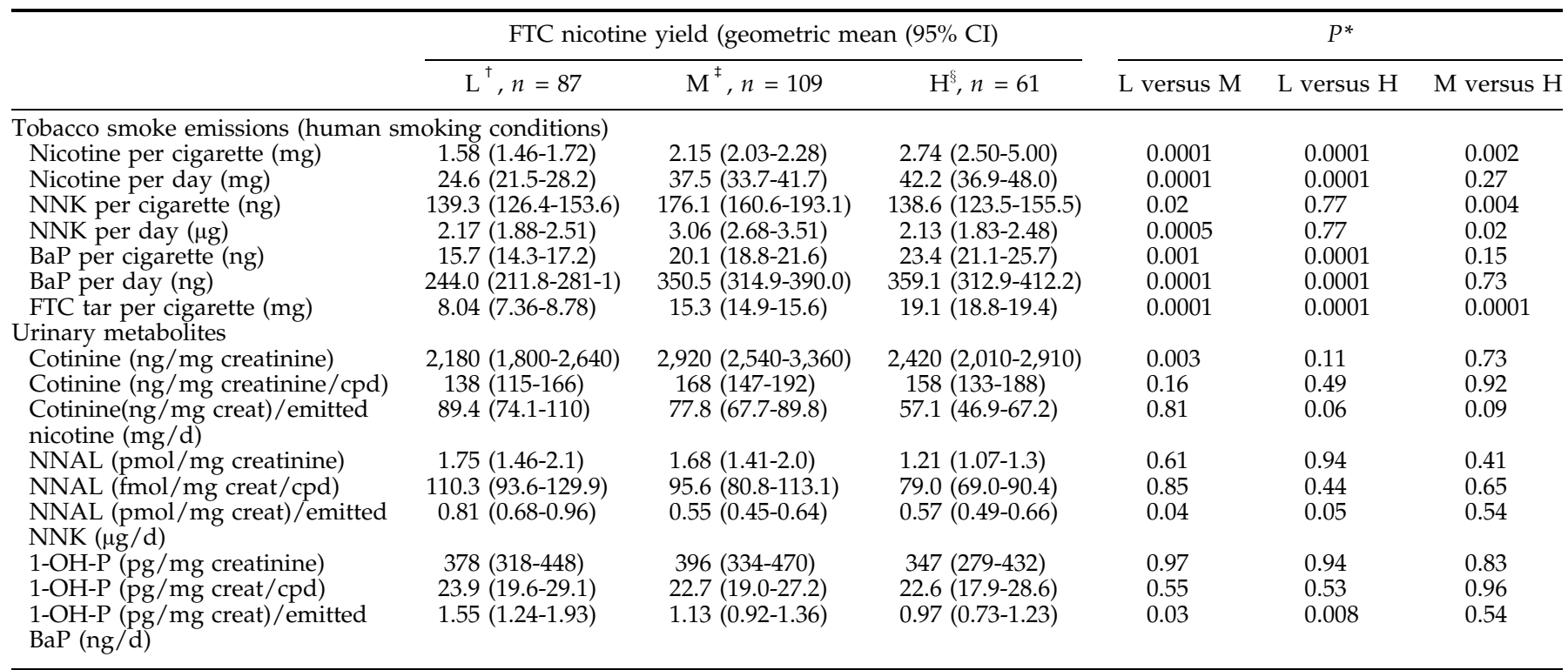

Abbreviation: cpd, cigarettes per day.

${ }^{*} P$ values were adjusted for sex, race, and BMI. Pairwise comparisons were based on Tukey multiple comparison procedure.

tLow-yield FTC nicotine group: range, $0.1-0.8 \mathrm{mg} /$ cigarette; mean, $0.66 \mathrm{mg} /$ cigarette; and median, $0.65 \mathrm{mg} /$ cigarette.

¥Medium-yield FTC nicotine group: range, $0.9-1.2 \mathrm{mg} /$ cigarette; mean, $1.12 \mathrm{mg} /$ cigarette; and median, $1.1 \mathrm{mg} /$ cigarette.

\$High-yield cigarette FTC nicotine group: range, 1.3-1.9 mg/cigarette; mean, $1.41 \mathrm{mg} /$ cigarette; and median, $1.4 \mathrm{mg} /$ cigarette. 
A

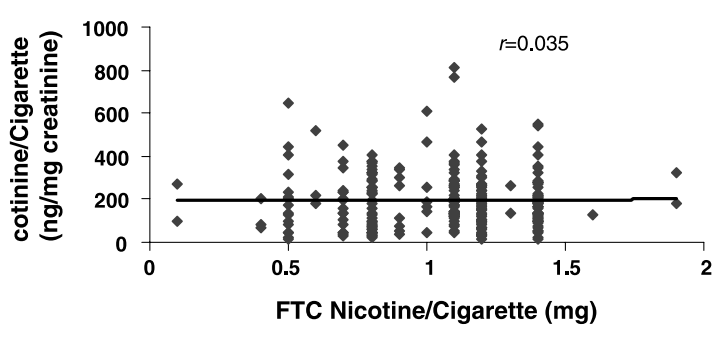

B

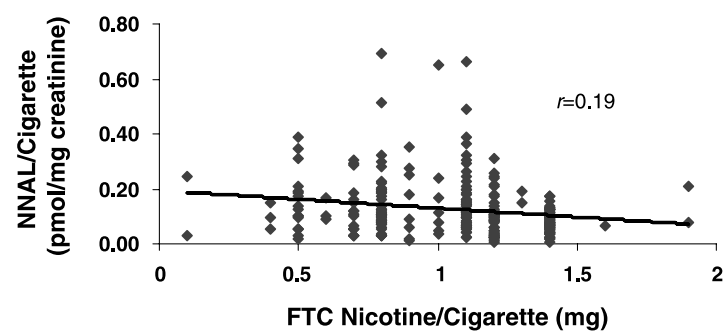

C

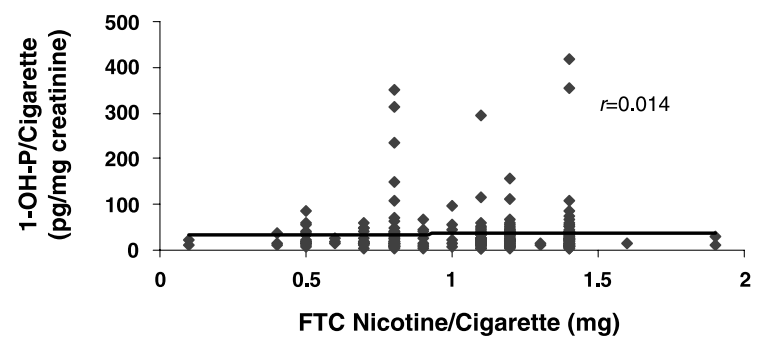

Figure 1. Relationship between nicotine, measured by the FTC method, and levels of urinary cotinine (A), total NNAL (B), and 1-OH-P $(\mathbf{C})$, all expressed per milligrams $(\mathrm{mg})$ creatinine in smokers' urine $(n=257)$.

there were significant differences in urinary metabolites per unit of delivered dose of parent toxins between smokers who smoked low- versus high-yield cigarettes (Table 3). Plots of FTC nicotine content versus levels of urinary cotinine, NNAL, and 1-OH-P per cigarette as shown in Fig. $1 \mathrm{~A}$ to $\mathrm{C}$, respectively, indicate that there is substantial interindividual variation in the excretion of cotinine, NNAL, and 1-OH-P among those who smoked cigarettes containing the same amount of nicotine according to FTC-mandated machine smoking parameters.

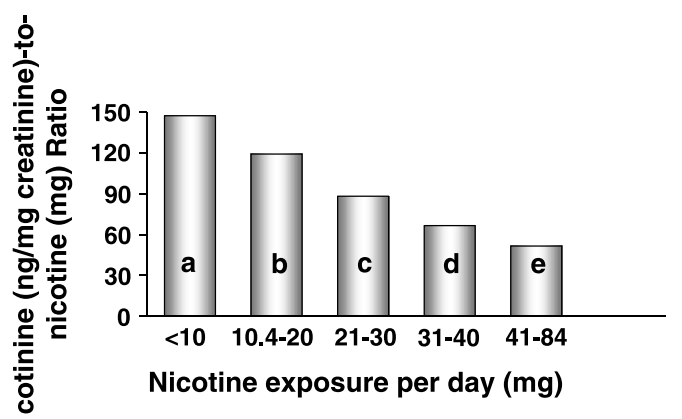

Figure 2. Means of cotinine-to-nicotine ratios in smokers for whom daily delivered dose of nicotine from cigarettes are $(a)<10 \mathrm{mg}(n=$ 46); (b) 10.4 to $20 \mathrm{mg}[n=118 ; P=0.06$ ( $a$ versus $b)]$; $(c) 21$ to 30 $\operatorname{mg}[n=61 ; P=0.001(a$ versus $c)$ and $P=0.01(b$ versus $c)] ;(d) 31$ to $40 \mathrm{mg}[n=19 ; P<0.0001(a$ versus $d)$ and $P=0.07(c$ versus $d)]$; and $(e) 41$ to $84 \mathrm{mg}[n=13 ; P<0.0001(a$ versus $e)$ and $P=0.1$ $(d$ versus $e)$ ].
A

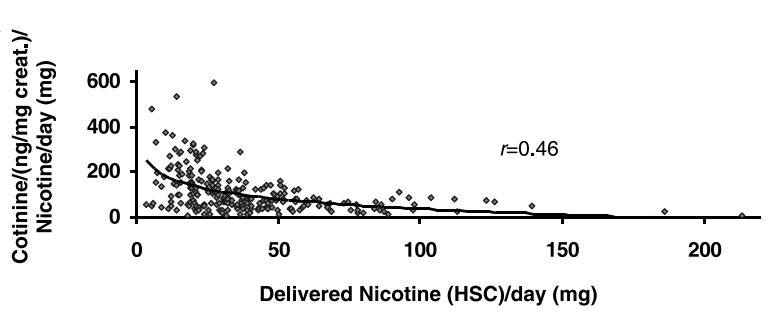

B

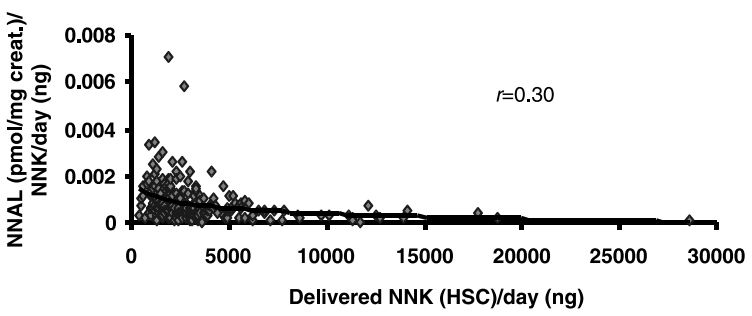

C

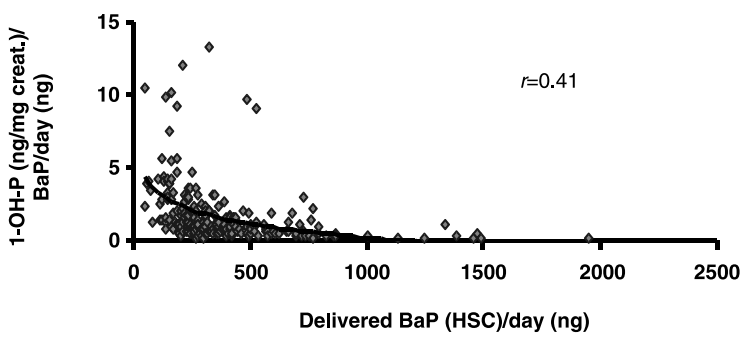

Figure 3. Relationship between daily delivered emissions of select tobacco smoke toxins as determined by machine smoking mimicking human smoking conditions (HSC) and corresponding urinary metabolites per unit of daily emissions of parent toxin in smokers. A. Cotinine/nicotine versus nicotine. B. NNAL/NNK versus NNK. C. 1-OH-P/BaP versus BaP. $r$, logarithmic regression.

Correlations between levels of FTC nicotine and each of the three urinary biomarkers were small ( $r=0.014-0.19)$ and none was statistically significant.

Relationships between Delivered Doses of Cigarette Smoke Toxins and Levels of Urinary Metabolites after Adjustment to Toxin Exposures. Figure 2 displays mean levels of cotinine after adjusting for creatinine and daily delivered nicotine (under human smoking conditions) in five subgroups that are exposed to different levels of nicotine per day. The results clearly show that with increasing daily delivered doses of nicotine, the excretion of cotinine per unit of nicotine emission decreases. Similarly, the cotinine-tonicotine ratio in smokers who smoked $\leq 20$ cigarettes per day $[118.4 \pm 95.4 \mathrm{ng} / \mathrm{mg}$ creatinine $/ \mathrm{mg} / \mathrm{d}$ (mean $\pm \mathrm{SD}) ; n=214)]$ was 3.5 -fold greater than those who smoked $>20$ cigarettes per day $(33.8 \pm 39.4 ; n=43 ; P=0.0005)$.

Plots of urinary cotinine, NNAL, and 1-OH-P per unit of delivered dose of the corresponding parent compound versus assessed individual exposures to nicotine, NNK, and $\mathrm{BaP}$, respectively, are shown in Fig. 3A to $\mathrm{C}$. With increasing amounts of delivered dose of parent compound, the level of urinary biomarker per unit of exposure (ratio of urinary metabolite-to-delivered dose) decreased for all biomarkers tested (Fig. 3A-C). A similar trend was observed in smokers who smoked the same brand of cigarettes, namely Marlboro (Fig. 4), with vented filter tip, as well as in smokers who smoked Newport cigarettes that have no vented filter tip but contain menthol (data not shown). Figure 4A shows levels of daily delivered nicotine from mainstream smoke and urinary cotinine for each Marlboro smoker. Fig. 4B (inset) shows a negative correlation between cotinine-to-nicotine ratios and 
daily delivered doses of nicotine in subgroups of smokers who smoked Marlboro ( $r=-0.59$; linear regression) and corresponding $r$ value for Newport was -0.37 (data not shown).

\section{Discussion}

A great body of evidence suggests that yields per cigarette of tar, nicotine, and other smoke constituents, derived from machine smoking with FTC/International Standards Organization protocols, do not provide valid estimates of human exposure and risk for tobacco-related disease (National Cancer Institute Monograph No. 13). Urinary metabolites of tobacco smoke toxins were widely used as biomarkers for the assessment of direct and passive exposure to cigarette smoke $(8,10,11)$. Urinary metabolites are generally affected by interindividual differences in metabolism; the intensity of exposure may also modify the metabolism through altered expression of relevant enzymes.

Levels of urinary cotinine, total NNAL, and 1-OH-P were not significantly different in smokers who smoked low-yield cigarettes, as ranked by the FTC machine smoking method, by comparison to those who smoked high-yield cigarettes (Fig. 1; Table 3), which are consistent with previous findings (8). However, there were significant differences in delivered doses of nicotine, NNK, and $\mathrm{BaP}$ from mainstream smoke when smokers' customary cigarettes were machine smoked under conditions matching the habits of each subject in the study. Delivered dosages of nicotine and $\mathrm{BaP}$ in subjects who smoked low-yield cigarettes were lower than those who smoked highyield cigarettes. Hence, smokers of low-yield cigarettes excrete more metabolites per delivered dosage of toxins than those who smoke high-yield cigarettes (Table 3). The mean ratios of cotinine-to-nicotine, NNAL-to-NNK, and 1-OH-P-to-BaP were $64 \%, 70 \%$, and $62 \%$ higher, respectively, in smokers of lowyield cigarettes than in those who smoked high-yield cigarettes (Table 3) and the ratios of metabolites-to-parent compounds decreased with increasing delivered doses of the parent toxin (Figs. 2, 3, and 4B).

Smokers' exposure to toxins is affected by the type/brand of cigarettes used, individual smoking behavior, and by the number of cigarettes smoked per day. To adjust the role of cigarette design factors on interindividual variability of urinary biomarkers, we compared relationships of delivered doses of carcinogens and urinary metabolites among smokers who smoked the same brand of cigarettes, namely Marlboro, a popular brand of cigarette in the United States that features ventilation holes in its filter tip and is smoked mostly by EuroAmericans. Again, an inverse relationship was observed between cotinine-to-nicotine ratios and delivered daily doses of nicotine (Fig. 4B, inset). Filter vents are designed to dilute mainstream smoke and produce less smoke in the burning cone that changes the chemical composition of the combustion product (5). Some smokers block filter vents with either fingertips or lips; exposure to cigarette smoke toxins increases slightly when filter vents are blocked. Furthermore, to eliminate the filter vent blocking factor, we investigated another popular brand of cigarette that does not have filter ventilation, namely the mentholated Newport that is smoked mostly by African-Americans, and we found that the same trend persists; the intake, conversion, and excretion of toxins to urinary metabolites decrease with increased delivered dosage (data not shown).

Smokers who inhaled 10 to $20 \mathrm{mg}$ nicotine/d excreted about twice as much cotinine per unit of nicotine than those who inhaled 31 to $40 \mathrm{mg}$ nicotine/d (Fig. 2). The current study indicates that increasing the number of cigarettes smoked per day results in a decreased ratio of cotinine-to-nicotine; the mean of cotinine per unit of nicotine in the subgroup smoking $\leq 20$ cigarettes per day was 3.5-fold higher than that in those who smoked $>20$ cigarettes per day.

Our findings suggest that a higher degree of exposure may also alter intake and metabolism of other tobacco smoke toxins and excretion of urinary metabolites. There are many

A

Marlboro Cigarettes
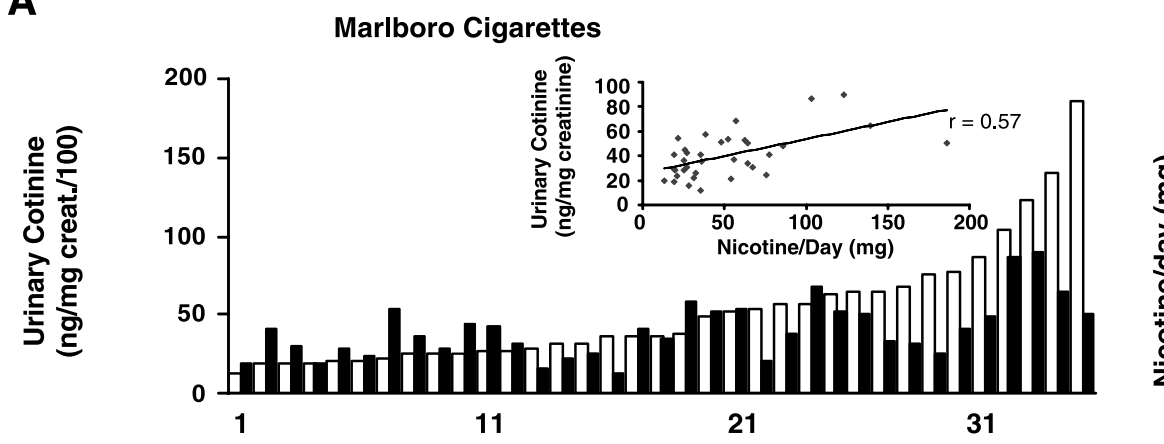

Figure 4. A. Levels of daily delivered nicotine in smokers who smoked Marlboro (1.1 mg FTC nicotine/cigarette; white columns) and urinary cotinine $/ 100$ (black columns). Inset, the relationship between daily nicotine emissions from cigarettes and excretion of urinary cotinine. B. Levels of daily delivered nicotine (white columns) and the ratios of cotinine-to-daily delivered dosage of nicotine (black columns).
B
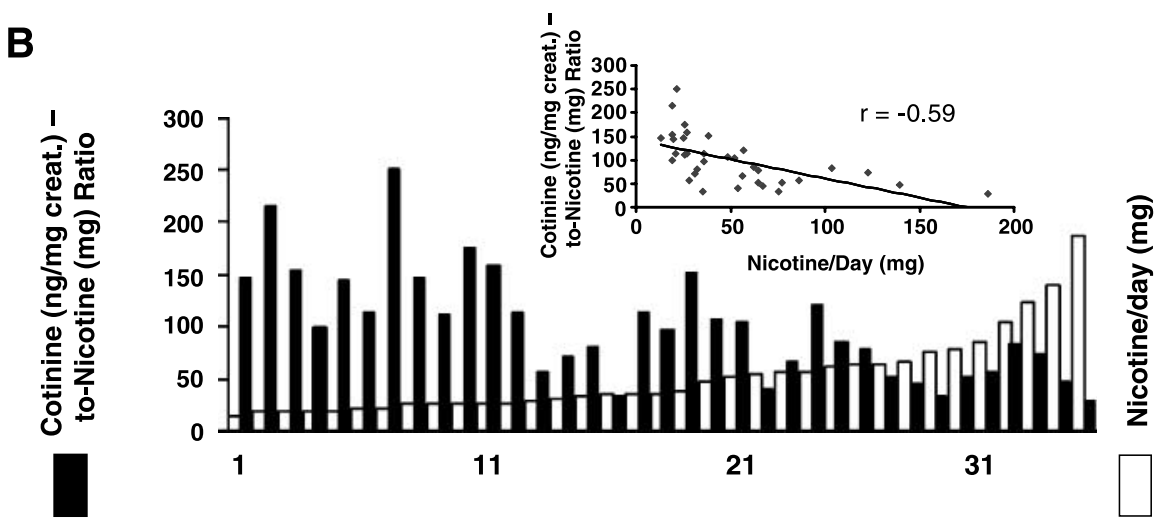

Subject Number 
compounds in tobacco smoke that could alter the metabolism of nicotine and other carcinogens and down-regulate several enzyme activities. Benowitz and Jacob (22) have shown that nicotine metabolism is decreased in smokers versus nonsmokers. Similarly, Lee et al. (23) have shown that smokers have slower nicotine clearance after an overnight abstinence period compared with a 7-day abstinence period. Schoedel et al. (24) have shown that long-term, in vivo nicotine treatment of African green monkeys decreased in vitro nicotine metabolism and the expression of hepatic CYP2A6 protein. CYP2A6 is a primary enzyme that activates nicotine to cotinine, as well as NNK to NNAL, suggesting that nicotine may decrease its own metabolism by decreasing expression of the nicotinemetabolizing enzyme CYP2A6. Thus, nicotine or other constituents of tobacco smoke may change the metabolism or pharmacokinetics with increasing nicotine or carcinogen intake at higher intensity of tobacco smoke exposure.

The current findings agree with other published studies. Law et al. (25) found that the ratio of serum hemoglobin to number of cigarettes smoked per day decreased with increasing smoking intensity. Vineis et al. $(26,27)$ have found that the dose-response for cancers of the lung and bladder is leveling off in heavy smokers who have regularly consumed 20 to 40 cigarettes per day. This apparent dose-response "ceiling" was originally reported for bladder cancer by Wynder and Stellman (28). In a large case-control study of lung cancers using a novel exposure rate model, Lubin and Caporaso (29) have shown a direct intensity rate effect at low smoking intensities and an inverse intensity rate effect at higher intensities. Haiman et al. (30) have found significant differences in the association between cigarette smoking and the risk of lung cancer among five self-reporting ethnic and racial populations. These differences were not observed among heavy smokers ( $>30$ cigarettes per day).

Our observation suggests that a slight reduction of the number of cigarettes smoked per day or changing type of cigarettes to those with a slightly lower yield may affect and slightly increase the overall intake of carcinogens and/or metabolism and thus will not have much potential to reduce toxic metabolites so as to produce a significant health effect. Indeed, recent assessments of morbidity and mortality suggest that low-yield products are associated with far less health benefit (31).

The way each cigarette is smoked by an individual governs mainstream smoke yields and, consequently, the smoker's exposure to harmful compounds. Our studies have shown that interindividual variation of carcinogen intake from mainstream smoke differs by $\sim 4$-fold due to smoking behavior $(7,32,33)$.

There were some limitations in the current PAH study. BaP was used as a biomarker of exposure to PAHs, whereas $1-\mathrm{OH}-$ $\mathrm{P}$, a metabolite of pyrene, was used as a biomarker of $\mathrm{PAH}$ exposures. The reason for this substitution is that the concentrations of $\mathrm{BaP}$ metabolites in urine are low so that they are not sufficiently sensitive as a biomarker of $\mathrm{PAH}$ exposure (34). By contrast, pyrene, which is a dominant compound in the PAH mixture at a concentration of $\sim 50$ to $270 \mathrm{ng} /$ cigarette in mainstream smoke, is mainly metabolized to the intermediary $1-\mathrm{OH}-\mathrm{P}$, which forms predominantly 1$\mathrm{OH}-\mathrm{P}$-gluc and, to much smaller extent, a sulfate conjugate, all of which are excreted in urine (35). Because the introduction of the urinary 1-OH-P as a biomarker of human PAH exposure, many studies have confirmed that $1-\mathrm{OH}-\mathrm{P}$ is a valid and sensitive indicator of PAH exposure $(36,37)$. The correlation between $\mathrm{BaP}$ exposure from mainstream cigarette smoke and urinary $1-\mathrm{OH}-\mathrm{P}$ in the current study is $r=0.48(n=231)$.

In conclusion, our data indicate that delivered doses of toxins from mainstream smoke affect the intake, metabolism, and excretion of metabolites in urine; at lower exposure, more metabolites appear in the urine than at higher exposures per unit of exposure. Thus, when urinary metabolites are used as biomarkers of exposure to toxic compounds of cigarette smoke, several factors, such as interindividual variation in metabolism and intensity of exposure, and other factors that are not presented in this paper, such as menthol-presenting cigarettes and gender (32), need to be taken in consideration.

\section{Acknowledgments}

We thank Julie Heidbrink, Alicia Czander, and Irene Delgado for recruiting smokers; Mohamed Tika for data management; James Hosey and Jie Zang for assistance with the analysis of cigarette smoke emissions and urinary metabolites; and Stephen Colosimo for cotinine and creatinine analyses.

\section{References}

1. International Agency for Research on Cancer. Tobacco smoke and involuntary smoking. IARC monographs on the evaluation of carcinogenic risks of chemicals to humans, vol. 83. Lyon (France): IARC; 2004. pp. 1-1438

2. U.S. Department of Health and Human Services. The health consequences of smoking: a report of the surgeon general. U.S. Department of Health and Human Services, Centers for Disease Control and Prevention, National Smoking and Health, Superintendent of Documents. Washington (DC): U.S. Government Printing Office; 2004.

3. Bridges RB, Combs JG, Hamble JW, Turbek JA, Rehm SR, Haley NJ. Puffing topography as a determinant of smoke exposure. Pharmacol Biochem Behav 1990;37:29-39.

4. Hofer I, Nil R, Batting K. Nicotine yield as determinant of smoke exposure indicators and puffing behavior. Pharmacol Biochem Behav 1991;40:139-49.

5. Kozlowski LT, O'Connor RJ. Cigarette filter ventilation is a defective design because of misleading taste, bigger puffs, and blocked vents. Tob Control 2002;11 Suppl 1:140-50.

6. Djordjevic MV, Stellman SD, Zang E. Doses of nicotine and lung carcinogens delivered to cigarette smokers. J Natl Cancer Inst 2000;92: 106-11.

7. Melikian AA, Djordjevic MV, Hosey J, et al. Gender differences relative to smoking behavior and emissions of toxins from mainstream cigarette smoke. Nicotine Tob Res 2007;9:377-87

8. Hecht SS, Murphy SE, Carmella SG, et al. Similar uptake of lung carcinogens by smokers of regular, light, and ultralight cigarettes. Cancer Epidemiol Biomarkers Prev 2005;14:693-8.

9. Melikian AA, Hosey J, Zhang J, et al. Reduction of urinary metabolites of tobacco carcinogens in smokers who switched from conventional light cigarettes to a new cigarette with low levels of tobacco-specific nitrosamines and a modified filter tip [abstract 6414]. Proc Am Assoc Cancer Res 2003;44:1470.

10. Zedler BK, Kinser R, Oey J, et al. Biomarkers of exposure and potential harm in adult smokers of 3-7 mg tar yield (Federal Trade Commission) cigarettes and in adult non-smokers. Biomarkers 2006;11:201-20.

11. Feng $S$, Roethig HJ, Liang $Q$, et al. Evaluation of urinary 1-hydroxypyrene, $S$-phenylmercapturic acid, trans,trans-muconic acid, 3-methyladenine, 3ethyladenine, 8-hydroxy-2'-deoxyguanosine and thioethers as biomarkers of exposure to cigarette smoke. Biomarkers 2006;11:28-52

12. Shields PG. Tobacco smoking, harm reduction, and biomarkers. J Nat Cancer Inst 2002;94:1435-44.

13. Muscat J, Djordjevic MV, Stellman SD, Richie JP, Jr. Racial differences in exposure and glucuronidation of the tobacco-specific carcinogen 4-(methylnitrosamino)-1-(3-pyridyl)-butanone (NNK). Cancer 2005;103:1420-6.

14. Djordjevic MV, Fan J, Ferguson S, Hoffmann D. Self-regulation of smoking intensity. Smoke yields of the low-nicotine, low-'tar' cigarettes. Carcinogenesis 1995;16:2015-21.

15. Djordjevic MV, Hoffmann D, Hoffmann I. Nicotine regulates smoking patterns. Prev Med 1997;26:435-40.

16. Brunnemann KD, Hoffmann D. Analytical studies on tobacco-specific $N$-nitrosamines in tobacco and tobacco smoke. Crit Rev Toxicol 1991;21: 235-40.

17. Melikian AA, Sun P, Prokopczyk B, et al. Identification of benzo[a]pyrene metabolites in cervical mucus and DNA adducts in cervical tissues in humans by gas chromatography-mass spectrometry. Cancer Lett 1999;146: 127-34.

18. Singh R, Tucek M, Maxa K, Tenglerova J, Weyand EH. A rapid and simple method for the analysis of 1-hydroxypyrene glucuronide: a potential biomarker for polycyclic aromatic hydrocarbon exposure. Carcinogenesis 1995;16:2909-15.

19. Lee CK, Cho SH, Kang JW, et al. Comparison of three analytical methods for 1-hydroxypyrene glucuronide in urine after non-occupational exposure to polycyclic aromatic hydrocarbons. Toxicol Lett 1999;108:209-15.

20. Carmella SG, Akerkar S, Hecht SS. Metabolites of the tobacco-specific nitrosamine 4-(methylnitrosamino)-1-(3-pyridyl)-1-butanone in smokers' urine. Cancer Res 1993;53:721-4.

21. Melikian AA, Qu Q, Shore R, et al. Personal exposure to different levels of benzene and its relationships to the urinary metabolites $S$-phenylmercapturic acid and trans, trans-muconic acid. J Chromatogr B Analyt Technol Biomed Life Sci 2002;778:211-21. 
22. Benowitz NL, Jacob P 3rd. Effects of cigarette smoking and carbon monoxide on nicotine and cotinine metabolism. Clin Pharmacol Ther 2000;67:653-9.

23. Lee BL, Benowitz NL, Jacob P 3rd. Influence of tobacco abstinence on the disposition kinetics and effects of nicotine. Clin Pharmacol Ther 1987;41: 474-9.

24. Schoedel KA, Sellers EM, Palmour R, Tyndale RF. Down-regulation of hepatic nicotine metabolism and a CYP2A6-like enzyme in African green monkeys after long-term nicotine administration. Mol Pharmacol 2003;63: 96-104.

25. Law MR, Morris JK, Watt HC, Wald NJ. The dose-response relationship between cigarette consumption, biochemical markers, and risk of lung cancer. Br J Cancer 1997;75:1690-3.

26. Vineis $\mathrm{P}$, Alavanja M, Garte $\mathrm{S}$. Dose-response relationship in tobacco-related cancers of bladder and lung: a biochemical interpretation. Int J Cancer 2004 108:2-7.

27. Vineis P, Kogevinas M, Simonato L, Brennan P, Boffetta P. Levelling-off of the risk of lung and bladder cancer in heavy smokers: an analysis based on multicentric case-control studies and a metabolic interpretation. Mutat Res 2000;463:103-10.

28. Wynder EL, Stellman SD. The comparative epidemiology of tobacco-related cancers. Cancer Res 1977;37:4608-22.

29. Lubin JH, Caporaso NE. Cigarette smoking and lung cancer: modeling total exposure and intensity. Cancer Epid Biomarkers Prev 2006;15:517-23.

30. Haiman CA, Stram DO, Wilkens LR, et al. Ethnic and racial differences in the smoking-related risk of lung cancer. N Engl J Med 2006;354:333-42.
31. Harris JE, Thun MJ, Mondul AM. Cigarette tar yields in relation to mortality from lung cancer in cancer prevention study II prospective cohort, 1982-8 BMJ 2004;328:72-9.

32. Melikian AA, Djordjevic MV. The role of smoking topography in assessing human smoking and its utility for information machine smoking protocols. 7th Scientific Advisory Study Group on Tobacco Product Regulations (formerly known as SACTob) meeting. Montebello (Canada); September 2004

33. Melikian AA, Djordjevic MV, Stellman SD. Inter-individual variability of exposure to cigarette smoke toxins due to differences in smoking behavior and metabolism [abstract 39]. Proceedings of $12^{\text {th }}$ Annual Meeting of Society for Research on Nicotine and Tobacco (SNRT), 2006.

34. Simpson CD, Wu MT, Christiani DC, Santella RM, Carmella SG, Hecht SS Determination of $\mathrm{r}-7, \mathrm{t}-8,9, \mathrm{c}-10$-tetrahydroxy-7,8,9,10-tetrahydrobenzo[ $[a]$ pyrrene in human urine by gas chromatography/negative ion chemical ionization/mass spectrometry. Chem Res Toxicol 2000;13:271-80.

35. Jongeneelen FJ. Methods for routine biological monitoring of carcinogenic PAH-mixtures. Sci Total Environ 1997;199:141-9.

36. Tsai HT, Wu MT, Hauser R, et al. Exposure to environmental tobacco smoke and urinary 1-hydroxypyrene levels in preschool children. Kaohsiung J Med Sci 2003;19:97-104.

37. Melikian AA, Malpure S, John A, et al. Determination of hemoglobin and serum albumin adducts of benzo[ $a$ ]pyrene by gas chromatography-mass spectrometry in humans and their relation to exposure and to other biological markers. Polycyclic Aromatic Compounds 1999;17:125-34. 